Pacific Journal of Mathematics

REMARKS ON SINGULAR ELLIPTIC THEORY FOR
COMPLETE RIEMANNIAN MANIFOLDS 


\title{
REMARKS ON SINGULAR ELLIPTIC THEORY FOR COMPLETE RIEMANNIAN MANIFOLDS
}

\author{
H. O. Cordes And R. C. McOwen
}

This paper is about a $C^{*}$-algebra $\mathfrak{A}$ of 0-order pseudo-differential operators on $L^{2}(\Omega)$, where $\Omega$ is a complete Riemannian manifold which need not be compact. This algebra is designed to handle singular elliptic theory for certain $N$ th-order differential operators. In particular, this paper studies the maximal ideal space $M$ of the (commutative) algebra $\mathfrak{N} / \mathscr{K}$, where $\mathscr{K}$ denotes the compact ideal. The space $M$ contains the bundle of cospheres as a subspace, and in general will contain additional points at infinity of the manifold. The significance of this for elliptic theory lies in the fact that an operator $A \in \mathfrak{Y}$ is Fredholm if and only if the associated continuous function $\sigma_{A} \in C(M)$ is never zero.

1. Introduction. Let $\Omega$ be an $n$-dimensional paracompact $C^{\infty}$-manifold with complete Riemannian metric $d s^{2}=g_{i j} d x^{i} d x^{\prime}$ and surface measure $d \mu=\sqrt{ } g d x$ where $g=\operatorname{det}\left(g_{i j}\right)$. As in [5] we define $\Lambda=(1-\Delta)^{-1 / 2}$ as a positive-definite operator in $\mathscr{L}(\mathfrak{f})$, the bounded operators over the Hilbert space $\mathfrak{f}=L^{2}(\Omega, d \mu)$, and define the Sobolev spaces $\mathfrak{\mathfrak { f }}_{N} \subset \mathfrak{f}$ for $N=0,1, \cdots$ by requiring $\Lambda^{N}: \mathfrak{i} \rightarrow \mathfrak{f}_{N}$ to be an isometric isomorphism. It was shown in [3] that $C_{0}^{\infty}(\Omega)$ is then dense in each $\mathfrak{f}_{N}$.

In [5] we defined classes of bounded functions and vector fields, $\mathbf{A}$ and $\mathbf{D}$, whose successive covariant derivatives with respect to a symmetric affine connection $\nabla$ vanish at infinity in the special sense that for $f \in C(\Omega)$ we write $\lim _{x \rightarrow \infty} f=0$ if for every $\epsilon>0$ there exists a compact set $K \subset \Omega$ such that

$$
|f(x)|<\epsilon \quad \text { for } \quad x \in \Omega \backslash K \text {. }
$$

Let $\mathbf{L}^{N}$ denote the class of $N$ th-order differential operators generated by taking sums of products of elements in $\mathbf{D}$ and $\mathbf{A}$. The connection $\nabla$ need not be the Riemannian connection $\nabla g$, but must satisfy Condition $\left(r_{0}\right)$ of [5] that it does not differ drastically from $\nabla g$ at infinity. We also require Condition $\left(\mathbf{L}^{2}\right)$ that 1- $\Delta \in \mathbf{L}^{2}$, a condition which was seen in [5] to imply the curvature tensor $R$ tends to zero as $x \rightarrow \infty$ in the sense of (1.1). Under these two conditions it was shown that the operators $L \Lambda^{N}$ and $\Lambda^{N} L$ for $L \in \mathbf{L}^{N}$ are bounded over $\mathfrak{f}$ and thus generate an algebra $\mathfrak{U}^{0} \subset$ $\mathscr{L}(\mathfrak{F})$. Moreover it was found that after adding the compact ideal $\mathscr{K}$ to 
$\mathfrak{U}^{0}$ and taking the norm closure, we obtain a $C^{*}$-algebra $\mathfrak{U}$ with compact commutators.

In this paper we focus our attention on the maximal ideal space $\mathbf{M}$ of the commutative $C^{*}$-algebra $\mathfrak{A} / \mathscr{K}$. If we define the symbol $\sigma_{A}$ to be the continuous function on $\mathbf{M}$ associated with the coset $A+\mathscr{K}$, then a necessary and sufficient condition for $A$ to be Fredholm is that $\sigma_{A}$ never vanish on $\mathbf{M}$ (c.f. [1]). Thus a further analysis of $\mathbf{M}$ and the symbols $\sigma_{A}$ is desirable for the Fredholm theory of differential operators in $\mathbf{L}^{N}$. For compact manifolds $\Omega$ it was shown in [8] that $\mathbf{M}$ is just the bundle of unit co-spheres $S^{*} \Omega \subset T^{*} \Omega$. For the special noncompact manifold $\Omega=\mathbf{R}^{n}$ it was shown in [4] that $\mathbf{M}$ contains $S^{*} \Omega=\mathbf{R}^{n} \times S^{n-1}$ as a proper subset: in fact $\mathbf{M}=\partial P^{*} \Omega=P^{*} \Omega \backslash T^{*} \Omega$ where $P^{*} \Omega$ is a certain compactification of $T^{*} \Omega$. In both [4] and [8] explicit formulas for $\sigma_{A}$ were obtained. For general $\Omega$, the main result of this paper (c.f. Theorem 2.2) asserts the inclusions $S^{*} \Omega \subset \mathbf{M} \subset \partial P^{*} \Omega$. Although we do not achieve a complete description of $\mathbf{M}$ and $\sigma_{A}$, this theorem yields many results (e.g. criteria for "weak = strong" and characterizations of Fredholm essential spectra) of classical elliptic theory (c.f. [2]). For example, if $L \in \mathbf{L}^{N}$ is uniformly elliptic (see $\$ 2$ ) and formally self-adjoint, then $L$ is essentially self-adjoint (with domain $C_{0}^{\infty}(\Omega)$ ). A discussion of this and further applications of the result of this paper is planned for a subsequent publication.

2. The formal algebra symbol. Let $\mathfrak{A}_{M}$ denote the function algebra obtained by closing $\mathbf{A}$ under uniform norm. Since $\mathfrak{A}_{M}$ is a subalgebra of the bounded continuous functions on $\Omega$, the Gelfand isomorphism yields $\mathfrak{A}_{M} \cong C(\bar{\Omega})$ where $\bar{\Omega}$ is some compactification of $\Omega$. On the other hand, considering $\mathfrak{U}_{M} \subset \mathfrak{A}$ we obtain a canonical injection $i: \mathfrak{A}_{M} \rightarrow \mathfrak{A} / \mathscr{K}$ whose associated dual map $p=i^{*}$ provides a continuous surjection $p: \mathbf{M} \rightarrow \bar{\Omega}$. Let us denote the open subset $p^{-1}(\Omega) \subset \mathbf{M}$ by $\mathbf{S}$. The following theorem, which is an immediate consequence of Theorem 2.2 although we state it first for purposes of exposition, extends the corresponding well-known result for compact $\Omega$.

THEOREM 2.1. Let $\pi: S^{*} \Omega \rightarrow \Omega$ denote the fibre bundle of unit cospheres $S^{*} \Omega \subset T^{*} \Omega$. There is a (surjective) homeomorphism $\theta: \mathbf{S} \rightarrow S^{*} \Omega$ such that $\pi \circ \theta=p$ on $\mathbf{S}$ and for $m \in \mathbf{S}$ and $\theta(m)=$ $(x, \xi) \in S^{*} \Omega$ we have

$$
\begin{array}{ll}
\sigma_{a}(m)=a(x) & \sigma_{D \Lambda}(m)=b^{j}(x) \xi_{j} \\
\sigma_{\Lambda}(m)=0 & \sigma_{K}(m)=0
\end{array}
$$

where $K \in \mathscr{K}, a \in \mathbf{A}$, and $D \in \mathbf{D}$ is given in local coordinates by $-i b^{j}\left(\partial / \partial x^{j}\right)$ and $\xi=\xi_{j} d x^{j}$. 
For $a \in \mathbf{A}$ and $D \in \mathbf{D}$ with local expression $-i b^{\prime}\left(\partial / \partial x^{\prime}\right)$, the following formal symbols define continuous functions on $T^{*} \Omega$.

$$
\begin{gathered}
\tilde{\sigma}_{a}(x, \xi)=a(x) \quad \tilde{\sigma}_{D}(x, \xi)=b^{\prime}(x) \xi_{J} \\
\tilde{\sigma}_{\Lambda}(x, \xi)=\left(1+|\xi|^{2}\right)^{-1 / 2}
\end{gathered}
$$

and we may extend algebraically to sums and products. In particular, the formal symbols $\tilde{\sigma}_{a}, \tilde{\sigma}_{D \Lambda}$, and $\tilde{\sigma}_{\Lambda}$ for $a \in \mathbf{A}$ and $D \in \mathbf{D}$ generate a $C^{*}$-algebra, $\mathfrak{A}_{\sigma}$, of continuous bounded functions on $T^{*} \Omega$. The maximal ideal space of $\mathfrak{U}_{\sigma}$ is a compactification, $P^{*} \Omega$, of $T^{*} \Omega$, and we define the boundary $\partial P^{*} \Omega=P^{*} \Omega \backslash T^{*} \Omega$. The associated dual map to the injection $\mathfrak{U}_{M} \rightarrow \mathfrak{A}_{\sigma}$ provides a surjection of $P^{*} \Omega$ onto $\bar{\Omega}$, and the restriction of this map to the boundary is denoted by $\pi: \partial P^{*} \Omega \rightarrow \bar{\Omega}$. Using (2.1) of [5], the formal symbols of $L \Lambda^{N}$ and $\Lambda^{N} L$ for $L \in \mathbf{L}^{N}$ defined by algebraic extension of (2.2) are unique when restricted to $\partial P^{*} \Omega$. Thus we are lead to defining the formal algebra symbol as the algebra homomorphism

$$
\dot{\sigma}: \mathfrak{A}^{0} \rightarrow C\left(\partial P^{*} \Omega\right)
$$

obtained by this restriction of $\tilde{\sigma}$.

It is evident that $S^{*} \Omega$ is homeomorphic to $\pi^{-1} \Omega$ by the map $(x, \xi) \rightarrow \lim _{r \rightarrow \infty}(x, r \xi) \in \partial P^{*} \Omega$. Theorem 2.1 may be interpreted as providing a continuous injection $\theta: \mathbf{S} \rightarrow \partial P^{*} \Omega$ such that

$$
\dot{\sigma}_{A}(\theta(m))=\sigma_{A}(m)
$$

for $m \in \mathbf{S}$ and operators $A=a$ or $A=D \Lambda$. The main result of this paper extends this formula as follows.

THEOREM 2.2. Under Conditions $\left(r_{0}\right)$ and $\left(\mathbf{L}^{2}\right)$, there exists a continuous injection $\theta: \mathbf{M} \rightarrow \partial P^{*} \Omega$ such that

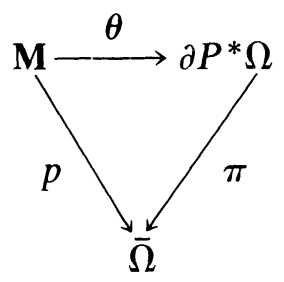

is commutative, surjective on fibres over $\Omega$, and (2.4) holds for all $m \in \mathbf{M}$ and $A \in \mathfrak{U}^{0}$.

If $L \in \mathrm{L}^{N}$ with $\dot{\sigma}_{L \Lambda^{N}}$ bounded away from zero on $S^{*} \Omega=\theta(\mathbf{S})$, we say $L$ is uniformly elliptic. 
3. Proof of Theorem 2.2. Condition $\left(\mathbf{L}^{2}\right)$ implies that we may write

$$
1-\Delta=\sum_{\nu=1}^{M} C_{\nu} D_{\nu}+\text { lower order terms }
$$

with $2 M$ vector fields $C_{\nu}, D_{\nu} \in \mathbf{D}$. Taking real and imaginary parts in (3.1), we may assume $C_{\nu}$ and $D_{v}$ are real. Let $B_{v} \in \mathbf{D}, \nu=1, \cdots, N$, be a basis for the module spanned by $C_{1}, \cdots, C_{M}, D_{1}, \cdots, D_{M}$ over the algebra of real-valued functions in A. In local coordinates, let $G$ denote the $n \times n$ matrix $\left(\left(g^{i j}\right)\right)$ and $B$ denote the $n \times N$ matrix $\left(\left(b_{v}^{i}\right)\right)$ where $b_{v}^{i}$ are the components of $B_{v}$. Let $B^{T}$ be the matrix transpose of $B$. Considering principal parts in (3.1), there is a symmetric $N \times N$ matrix-valued function $A=\left(\left(a_{\nu \mu}\right)\right)$ whose coefficients $a_{\nu \mu}$ are all real-valued functions of A, such that

$$
G=B A B^{T}
$$

Let us introduce the $N \times N$ matrix-valued function $P=B^{T} G^{-1} B A$. Observe that $P$ does not depend on local coordinates and $P^{2}=P$ implies that $P$ is a projection matrix with rank $n$. Let $\Gamma=\left(\begin{array}{l}N \\ n\end{array}\right)$, the binomial coefficient. We shall require the following lemma from linear algebra.

Lemma 3.1. For any $N \times N$ projection matrix $P$ with rank $n$, there exists an $n \times n$ diagonal matrix minor $\tilde{P}$ such that $|\operatorname{det} \tilde{P}| \geqq \Gamma^{-1}$.

Proof. Since $\operatorname{det}(P-\lambda)=(1-\lambda)^{n}(-\lambda)^{N-n}$, the coefficient of $\lambda^{N-n}$ is \pm 1 . But this coefficient equals the sum of all $n \times n$ diagonal minors. Since there are precisely $\Gamma$ such minors, at least one must have absolute value not less than $\Gamma^{-1}$.

Applying the lemma, we see that at each point $x \in \Omega$ there is a matrix minor $\tilde{P}$ of $P, \tilde{P}=B_{(\gamma)}^{\tau} G^{-1} B \tilde{A}$ where $B_{(\gamma)}$ denotes one of the $\Gamma$ distinct $n \times n$ matrix minors of $B$ and $\tilde{A}$ denotes a certain $N \times n$ matrix minor of $A$, such that

$$
|\operatorname{det} \tilde{P}|>(2 \Gamma)^{-1} \text {. }
$$

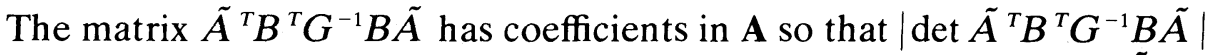
is uniformly bounded over $\Omega$. Thus $\left|\operatorname{det} G^{-1 / 2} B \tilde{A}\right|=$ $\left|\operatorname{det} \tilde{A}^{T} B^{T} G^{-1} B \tilde{A}\right|^{1 / 2}$ is also uniformly bounded. So by (3.3), there exists a constant $C>0$ such that at each $x \in \Omega$, $\left|\operatorname{det} B_{(\gamma)}^{T} G^{-1 / 2}\right|>C$ holds for at least one $\gamma=1, \cdots, \Gamma$. Observe that $d_{\gamma}=\operatorname{det} B_{(\gamma)}^{T} G^{-1 / 2}$ is a $C^{\infty}$ - 
function on $\Omega$ and we have a finite open cover of $\Omega$ by the $\Gamma$ sets

$$
\Omega_{\gamma}=\left\{x \in \Omega:\left|d_{\gamma}(x)\right|>C\right\} .
$$

Let us suppose that we have chosen $C$ such that also the sets

$$
\Omega_{\gamma}^{\prime \prime}=\left\{x \in \Omega:\left|d_{\gamma}(x)\right|>2 C\right\}
$$

cover $\Omega$. Also define

$$
\Omega_{\gamma}^{\prime}=\left\{x \in \Omega:\left|d_{\gamma}(x)\right|>\frac{4}{3} C\right\} .
$$

Observe that $\Omega_{\gamma}^{\prime \prime} \subset \Omega_{\gamma}^{\prime} \subset \Omega_{\gamma}$. Let $\overline{\Omega_{\gamma}^{\prime}}$ and $\overline{\Omega_{\gamma}^{\prime \prime}}$ denote the closures of $\Omega_{\gamma}^{\prime}$ and $\Omega_{\gamma}^{\prime \prime}$ respectively in $\bar{\Omega}$.

In each set $\Omega_{\gamma}$, det $B_{(\gamma)}>0$ so we may define the $n \times N$ matrix-valued function $Q_{\gamma}=B_{(\gamma)}^{-1} B$. Let us also define an $n \times n$ matrix-valued function on $\Omega_{\gamma}$

$$
A_{\gamma}=Q_{\gamma} A Q_{\gamma}^{T}=\left(B_{(\gamma)}^{-1} G^{1 / 2}\right)\left(G^{1 / 2} B_{(\gamma)}^{-1 T}\right)
$$

Clearly $A_{\gamma}$ is coordinate invariant and positive definite with spectrum bounded uniformly (over $\Omega_{\gamma}$ ) below by $\epsilon>0$. Since $\left|\operatorname{det} A_{\gamma}\right|<C^{-2}$ on $\Omega_{\gamma}$, we conclude that the spectrum of $A_{\gamma}$ is contained in a fixed (independent of $x \in \Omega_{\gamma}$ ) compact subset of $(0, \infty)$. Thus we may define $A_{\gamma}^{1 / 2}$ by a resolvent integral. A computation shows that the coefficients of $A_{\gamma}^{1 / 2}$ are bounded over $\Omega_{\gamma}$ and have covariant derivatives tending to zero in $\Omega_{\gamma}$ outside large compact sets of $\Omega$. Thus if we define $\tilde{B}_{(\gamma)}=$ $B_{(\gamma)} A^{1 / 2}$ we have $G=\tilde{B}_{(\gamma)} \tilde{B}_{(\gamma)}^{T}$ in $\Omega_{\gamma}$. In other words we have diagonalized the metric in $\Omega_{\gamma}$ as follows.

Proposition 3.2. Under Condition $\left(\mathbf{L}^{2}\right)$, there is a finite open cover of $\Omega$ by open sets $\left\{\Omega_{\gamma}\right\}_{\gamma=1}^{\prime}$ such that in each set $\Omega_{\gamma}$ we may express

$$
g^{l \prime}=\sum_{\nu=1}^{n} \tilde{b}_{\nu}^{\prime} \tilde{b}_{\nu}^{\prime}
$$

where the $n$ real vector fields $\tilde{B}_{\nu}$ with components $\tilde{b}_{\nu}^{i}$ are bounded over $\Omega_{\gamma}$ and satisfy: for every $n \geqq 1$ and $\epsilon>0$ there exists a compact set $K_{\epsilon} \subset \Omega$ such that

$$
\left|\nabla^{n} \tilde{B}_{\nu}\right|<\epsilon \quad \text { for all } \quad x \in \Omega_{\gamma} \backslash K_{\epsilon} .
$$

Now let $\chi \in C^{\infty}(\mathbf{R})$ with $\chi(t)=0$ for $t \leqq 0, \chi(t)=1$ for $t \geqq C$ and $0 \leqq \chi \leqq 1$ for $0<t$. Define $\varphi_{\gamma}(x)=\chi\left(3\left|d_{\gamma}(x)\right|-4 C\right), \quad \psi_{\gamma}(x)=$ 
$\chi\left(3\left|d_{\gamma}(x)\right|-3 C\right)$, and $\mu_{\gamma}(x)=\chi\left(3\left|d_{\gamma}(x)\right|-2 C\right)$. Dividing each $\varphi_{\gamma}$ by $\sum_{\gamma=1}^{\mathrm{r}} \varphi_{\gamma}$, we may assume $\sum_{\gamma=1}^{\mathrm{r}} \varphi_{\gamma} \equiv 1$ on $\Omega$. Observe $\varphi_{\gamma}, \psi_{\gamma}, \mu_{\gamma} \in \mathbf{A}, \varphi_{\gamma} \equiv 1$ on $\Omega_{\gamma}^{\prime \prime}$ and $\mu_{\gamma} \equiv 0$ on $\Omega \backslash \Omega_{\gamma}$. In fact $\psi_{\gamma} \equiv 1$ on $\overline{\Omega_{\gamma}^{\prime}}=\operatorname{supp} \varphi_{\gamma}$ and $\mu_{\gamma} \equiv 1$ on $\operatorname{supp} \psi_{\gamma}$, so $\varphi_{\gamma}=\varphi_{\gamma} \psi_{\gamma}$ and $\psi_{\gamma}=\psi_{\gamma} \mu_{\gamma}$. Observe that $D_{\gamma, \nu}=-i \mu_{\gamma} \tilde{B}_{\nu} \in \mathbf{D}$ (in particular, a vector field defined on all of $\Omega$ ).

Lemma 3.3. Vector fields of the form $\varphi_{\gamma} D$ and $\psi_{\gamma} D$ with $D \in \mathbf{D}$ may be written as $\varphi_{\gamma} D=\sum_{\nu=1}^{n} a_{\gamma, \nu} \varphi_{\gamma} D_{\gamma, \nu}$ and $\psi_{\gamma} D=\sum_{\nu=1}^{n} a_{\gamma, \nu} \psi_{\gamma} D_{\gamma, \nu}$ with $a_{\gamma, \nu} \in \mathbf{A}$.

Proof. If $D$ is given in local coordinates by $b^{j} \partial / \partial x^{j}$, simply define $a_{\gamma, \nu}=i b^{j} g_{j k} \tilde{b}_{\nu}^{k} \mu_{\gamma}$.

We now invoke some of the results of [5]. Condition $\left(r_{0}\right)$ implies that the formal adjoint of $\psi_{\gamma} D_{\gamma, \nu}$ is of the form $\left(\psi_{\gamma} D_{\gamma, \nu}\right)^{\prime}=\psi_{\gamma} D_{\gamma, \nu}+a$ with $\lim _{x \rightarrow \infty} a=0$ (c.f. (2.2) of [5]). Thus if we let $T_{\gamma, \nu}=\psi_{\gamma} D_{\gamma, \nu} \Lambda \in \mathfrak{A}^{0}$, we have by Remark 2.3, Theorem 3.1, and Proposition 4.4 of [5] that

$$
T_{\gamma, \nu}^{*}=\Lambda\left(\psi_{\gamma} D_{\gamma, \nu}\right)^{\prime} \equiv \Lambda \psi_{\gamma} D_{\gamma, \nu} \equiv \psi_{\gamma} D_{\gamma, \nu} \Lambda=T_{\gamma, \nu}(\bmod \mathscr{K}) .
$$

Also observe

$$
\begin{aligned}
\sum_{\nu=1}^{n}\left(\psi_{\gamma} D_{\gamma, \nu}\right)^{\prime}\left(\psi_{\gamma} D_{\gamma, \nu}\right) & =-\frac{1}{\sqrt{g}} \frac{\partial}{\partial x^{i}} g^{i j} \psi_{\gamma}^{2} \sqrt{g} \frac{\partial}{\partial x^{j}} \\
& =-\psi_{\gamma}^{2} \Delta-D
\end{aligned}
$$

with $\lim _{x \rightarrow \infty}|D|=0$. Thus using Corollary 3.6 of [5] together with (3.9) and (3.10) above

$$
\begin{array}{rlr}
\psi_{\gamma}^{2} & =\psi_{\gamma}^{2} \Lambda(1-\Delta) \Lambda \equiv\left(\psi_{\gamma} \Lambda\right)^{2}-\Lambda \psi_{\gamma}^{2} \Delta \Lambda & (\bmod \mathscr{K}) \\
& \equiv\left(\psi_{\gamma} \Lambda\right)^{2}-\sum_{\nu=1}^{n} \Lambda\left(\psi_{\gamma} D_{\gamma, \nu}\right)^{\prime}\left(\psi_{\gamma} D_{\gamma, \nu}\right) \Lambda & (\bmod \mathscr{K}) \\
& \equiv \sum_{\nu=0}^{n} T_{\gamma, \nu}^{2} \quad(\bmod \mathscr{K}) &
\end{array}
$$

where we have also defined $T_{\gamma, 0}=\psi_{\gamma} \Lambda$. Similarly, let us define $S_{\gamma, \nu}=$ $\varphi_{\gamma} T_{\gamma, \nu}$ for all $\gamma$ and $\nu$.

Let $\mathbf{M}_{\gamma}^{\prime}=p^{-1}\left(\overline{\Omega_{\gamma}^{\prime}}\right) \subset \mathbf{M}$. Also let $S^{n}$ be the half-sphere $\left\{\sigma=\left(\sigma_{0}, \sigma_{1}, \cdots, \sigma_{n}\right) \in \mathbf{R}^{n+1}: \sum_{\nu=0}^{n} \sigma_{\nu}^{2}=1 \quad\right.$ and $\left.\quad \sigma_{0} \geqq 0\right\}$, and $S^{n-1}=\partial S_{+}^{n}$.

Proposition 3.4. For each $\gamma=1, \cdots, \Gamma$ there is a continuous injection

$$
\mathbf{M}_{\gamma}^{\prime} \rightarrow \overline{\Omega_{\gamma}^{\prime}} \times S_{+}^{n}
$$


where $m \mapsto(x, \sigma)$ such that $\sigma_{a}(m)=a(x)$ and $\sigma_{S_{\gamma, \nu}}(m)=\varphi_{\gamma}(x) \sigma_{\nu}$ for $\nu=0,1, \cdots . n$. In addition, (3.12) maps $\mathbf{M}_{\gamma}^{\prime} \cap \mathbf{S}$ onto $\Omega_{\gamma}^{\prime} \times S^{n-1}$.

Proof. Let $\mathfrak{A}_{\gamma}^{*}$ denote the smallest $C^{*}$-algebra with unit containing $\mathscr{K}$ and $T_{\gamma, \nu}$ for $\nu=0,1, \cdots, n$. Let $\mathfrak{A}_{\gamma}$ denote the smallest $C^{*}$-algebra containing $\mathfrak{U}_{M}$ and $\mathfrak{U}_{\gamma}^{*}$. Since $\mathfrak{U}_{\gamma} / \mathscr{K}$ is a commutative $C^{*}$-algebra, let $\mathbf{N}_{\gamma}$ denote its maximal ideal space, and let $\sigma^{\gamma}: \mathfrak{A}_{\gamma} \rightarrow C\left(\mathbf{N}_{\gamma}\right)$ be the symbol homomorphism. Also let $p_{\gamma}: \mathbf{N}_{\gamma} \rightarrow \bar{\Omega}$ be the associated dual map to the inclusion $\mathfrak{A}_{M} \rightarrow \mathfrak{A}_{\gamma}$. For $a \in \mathbf{A}$ and $D \in \mathbf{D}$, define $\rho_{\gamma}(a)=a, \rho_{\gamma}(D \Lambda)=$ $\psi_{\gamma} D \Lambda$, and $\rho_{\gamma}(\Lambda)=\psi_{\gamma} \Lambda$. By Lemma 3.3, $\rho_{\gamma}$ extends to a continuous algebra homomorphism of $\mathfrak{A}$ onto $\mathfrak{A}_{\gamma}$. Since $\rho_{\gamma}(\mathscr{K}) \subset \mathscr{K}$, there is an induced surjective homomorphism $\bar{\rho}_{\gamma}: \mathfrak{A} / \mathscr{K} \rightarrow \mathfrak{A}_{\gamma} / \mathscr{K}$. Thus the associated dual map $i_{\gamma}=\bar{\rho}_{\gamma}^{*}$ provides a continuous injection such that

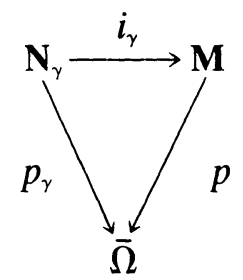

commutes and

$$
\sigma_{\rho_{\gamma}(A)}^{\gamma}(n)=\sigma_{A}\left(i_{\gamma} n\right)
$$

for all $A \in \mathfrak{U}$ and $n \in \mathbf{N}_{\gamma}$. The restriction of $i_{\gamma}$ to $\mathbf{N}_{\gamma}^{\prime}=p_{\gamma}^{-1}\left(\bar{\Omega}_{\gamma}^{\prime}\right)$ may easily be seen to provide a surjection of $\mathbf{N}_{\gamma}^{\prime}$ onto $\mathbf{M}_{\gamma}^{\prime}$. Thus we may consider $\mathbf{N}_{\gamma}^{\prime} \subset \mathbf{M}$.

On the other hand, $\mathfrak{U}_{\gamma}^{*} / \mathscr{K}$ is also a commutative $C^{*}$-algebra with unit whose maximal ideal space will be denoted $\mathbf{M}_{\gamma}^{*}$. But by a wellknown theorem concerning $C^{*}$-algebras generated by a finite number of elements (c.f. [7]), $\mathbf{M}_{\gamma}^{\#}$ is homeomorphic to the joint spectrum of the $n+1$ cosets $T_{\gamma, \nu}+\mathscr{K}$ of $\mathfrak{A}_{\gamma}^{*} / \mathscr{K}$. Using (3.11) and the non-negativity of $T_{\gamma, 0}$, this implies that $\mathbf{M}_{\gamma}^{*} \subset B_{+}^{n+1}=\left\{r \sigma: \sigma \in S_{+}^{n}\right.$ and $\left.0 \leqq r \leqq 1\right\}$. Since $\mathfrak{A}_{\gamma}$ is generated by $\mathfrak{U}_{M}$ and $\mathfrak{U}_{\gamma}^{*}$, Herman's Lemma (c.f. [6] Theorem 1) implies the existence of a continuous injection

$$
\mathbf{N}_{\gamma} \rightarrow \bar{\Omega} \times B_{+}^{n+1}
$$

such that $n \mapsto\left(x, \psi_{\gamma}(x) \sigma\right)$ where $\sigma_{a}^{\gamma}(n)=a(x)$ and $\sigma_{T_{\gamma, \nu}}^{\gamma}(n)=\psi_{\gamma}(x) \sigma_{\nu}$ for $\nu=0,1, \cdots, n$. But since $\psi_{\gamma} \equiv 1$ on $\overline{\Omega_{\gamma}^{\prime}}$, the image of $\mathbf{N}_{\gamma}^{\prime}$ under (3.15) is contained in $\overline{\Omega_{\gamma}^{\prime}} \times S_{+}^{n}$. Combining this with (3.13) and (3.14) yields (3.12) with $\sigma_{a}(m)=\sigma_{a}^{\gamma}\left(i_{\gamma}^{-1} m\right)=a(x)$ and $\sigma_{S_{\gamma, \nu}}(m)=$ $\sigma_{\varphi_{\gamma}}^{\gamma}\left(i_{\gamma}^{-1} m\right) \cdot \sigma_{T_{\gamma, \nu}}^{\gamma}\left(i_{\gamma}^{-1} m\right)=\varphi_{\gamma}(x) \sigma_{\nu}$. 
Finally, let $m^{1} \in \mathbf{M}_{\gamma}^{\prime}$ with $p\left(m^{1}\right)=x \in \Omega_{\gamma}^{\prime}$. Let $\varphi \in C_{0}^{\infty}\left(\Omega_{\gamma}^{\prime}\right)$ with $\varphi(x)=1$. Then $\sigma_{T_{\gamma, 0}}\left(m^{1}\right)=\sigma_{\varphi T_{\gamma, 0}}\left(m^{1}\right)=0$ since $\varphi T_{\gamma, 0}=\varphi \Lambda \in \mathscr{K} \quad$ by Theorem 3.1 of [5]. Thus $m^{1} \mapsto\left(x, \sigma^{1}\right)$ with $\sigma^{1} \in S^{n-1}$. Let $\sigma^{2}$ be arbitrary in $S^{n-1}$ and $0=\left(\left(r_{\nu \mu}\right)\right)$ an orthogonal $n \times n$ matrix such that $\sigma^{2}=0 \sigma^{1}$. Defining $\tau(a)=a, \tau\left(T_{\gamma, 0}\right)=T_{\gamma, 0}, \tau\left(T_{\tau, \nu}\right)=\Sigma r_{\nu \mu} T_{\gamma, \mu}$ induces a surjective automorphism $\bar{\tau}: \mathfrak{A}_{\gamma} / \mathscr{K} \rightarrow \mathfrak{A}_{\gamma} / \mathscr{K}$. The associated dual map $\bar{\tau}^{*}: \mathbf{M}_{\gamma} \rightarrow \mathbf{M}_{\gamma}$ is a homeomorphism such that

$$
\sigma_{\tau(A)}(m)=\sigma_{A}\left(\bar{\tau}^{*} m\right)
$$

for all $A \in \mathfrak{A}_{\gamma}$ and $m \in \mathbf{M}_{\gamma}$. In particular, for $A=T_{\gamma, v}$ and $m^{2}=\bar{\tau}^{*} m^{1}$,

$$
\sigma_{T_{\gamma, \nu}}\left(m^{2}\right)=\sum r_{\nu \mu} \sigma_{T_{\gamma, \mu}}\left(m^{1}\right)=\sigma_{\nu}^{2}
$$

implies $m^{2} \mapsto\left(x, \sigma^{2}\right)$. Hence (3.12) provides a homeomorphism of $\mathbf{M}_{\gamma}^{\prime} \cap \mathbf{S}$ onto $\Omega_{\gamma}^{\prime} \times S^{n-1}$.

Let $\mathfrak{A}_{\sigma, \tau}$ denote the $C^{*}$-subalgebra of $C B\left(T^{*} \Omega_{\gamma}^{\prime}\right)$ obtained by restricting functions in $\mathfrak{A}_{\sigma}$ to $T^{*} \Omega_{\gamma}^{\prime}$. Let $P^{*} \Omega_{\gamma}^{\prime}$ denote the compactification of $T^{*} \Omega_{\gamma}^{\prime}$ induced by $\mathfrak{A}_{\sigma, \gamma}$, and consider the functions $\tilde{\sigma}_{A}$ extended to $P^{*} \Omega_{\gamma}^{\prime}$ without change in notation.

Proposition 3.5. For each $\gamma=1, \cdots, \Gamma$ there is a continuous injection

$$
P^{*} \Omega_{\gamma}^{\prime} \rightarrow \overline{\Omega_{\gamma}^{\prime}} \times S_{+}^{n}
$$

such that $(p) \mapsto(x, \sigma)$ with $\tilde{\sigma}_{a}(p)=a(x)$ and $\tilde{\sigma}_{S_{\gamma, \nu}}(p)=\varphi_{\gamma}(x) \sigma_{\nu}$ for $\nu=$ $0,1, \cdots n$. In fact (3.16) is surjective.

Proof. Restricting the formal symbols $\tilde{\sigma}_{T_{\gamma, \nu}}$ for $\nu=0,1, \cdots, n$ to $T^{*} \Omega_{\gamma}^{\prime}$ generates a $C^{*}$-algebra with maximal ideal space $S_{+}^{n}$ and which together with $\left.\mathfrak{A}_{M}\right|_{\Omega_{\gamma}^{\prime}}$ generates $\mathfrak{A}_{\sigma, \gamma}$. Herman's Lemma yields the injection (3.16) which may easily be seen to be surjective.

Now we may prove our main result.

Proof of Theorem 2.2. For each $\gamma=1, \cdots, \Gamma,(3.12)$ together with (3.16) yield a map $\theta_{\gamma}: \mathbf{M}_{\gamma}^{\prime} \rightarrow P^{*} \Omega_{\gamma}^{\prime}$ such that $\sigma_{a}(m)=\tilde{\sigma}_{a}\left(\theta_{\gamma}(m)\right)$ and $\sigma_{S_{\gamma, \nu}}(m)=\tilde{\sigma}_{S_{\gamma, \nu}}\left(\theta_{\gamma}(m)\right)$ for $\nu=0,1, \cdots, n$. Since each $P^{*} \Omega_{\gamma}^{\prime} \subset P^{*} \Omega$, and $m \in \mathbf{M}_{\gamma}^{\prime} \cap \mathbf{M}_{\delta}^{\prime}$ implies $\tilde{\sigma}_{S_{\gamma, \nu}}\left(\theta_{\gamma}(m)\right)=\tilde{\sigma}_{S_{\delta, \nu}}\left(\theta_{\delta}(m)\right)$ for every $\nu=0,1, \cdots, n$, the $\theta_{\gamma}$ induce a continuous injection $\theta: \mathbf{M} \rightarrow P^{*} \Omega$ such that

$$
\sigma_{A}(m)=\tilde{\sigma}_{A}(\theta(m))
$$


for all $A=a$ or $S_{\gamma, \nu}, a \in \mathbf{A}$ and $\gamma=1, \cdots, \Gamma$ and $\nu=0,1, \cdots, n$. Now let $D \in \mathrm{D}$, and write $D \Lambda=\Sigma_{\gamma} \varphi_{\gamma} D \Lambda=\sum_{\gamma, \nu} a_{\gamma, \nu} S_{\gamma, \nu}$ by Lemma 3.3. Then

$$
\sigma_{D \Lambda}(m)=\sum_{\gamma, \nu} \sigma_{a_{\gamma, \nu}}(m) \sigma_{S_{\gamma, \nu}}(m)=\sum_{\gamma, \nu} \bar{\sigma}_{a_{\gamma, \nu}}(\theta(m)) \tilde{\sigma}_{S_{\gamma, \nu}}(\theta(m))=\tilde{\sigma}_{D \Lambda}(\theta(m)) .
$$

Similarly $\sigma_{\Lambda}(m)=\tilde{\sigma}_{\Lambda}(\theta(m))$. In fact the second statement of Proposition 3.4 implies that the image of $\theta$ is contained in $\partial P^{*} \Omega$, and (3.17) becomes

$$
\sigma_{A}(m)=\dot{\sigma}_{A}(\theta(m))
$$

for all $A=a, D \Lambda$, or $\Lambda$ with $a \in \mathbf{A}$ and $D \in \mathbf{D}$. The extension of (3.18) to all $A \in \mathfrak{A}^{0}$ follows from the algebraic properties of $\sigma$ and $\tilde{\sigma}$.

\section{REFERENCES}

1. M. Breuer and H. O. Cordes, On Banach algebras with $\sigma$-symbol, J. Math. Mech., 13 (1964), 313-324.

2. F. Browder, On the spectral theory of elliptic differential operators I, Math. Ann., 142 (1961), 22-130.

3. H. O. Cordes, Self-adjointness of powers of elliptic operators on noncompact manifolds, Math. Ann., 195 (1972), 257-272.

4. H. O. Cordes and E. Herman, Gel'fand theory of pseudo-differential operators, Amer. J. Math., 90 (1968), 681-717.

5. H. O. Cordes and R. C. McOwen, The $C^{*}$-algebra of a singular elliptic problem on a noncompact Riemannian manifold, Math. Z., 153 (1977), 101-116.

6. E. Herman, The symbol of the algebra of singular integral operators, J. Math. Mech., 15 (1966), 147-156.

7. C. E. Rickart, General theory of Banach algebras, v. Nostrand, Princeton, 1960.

8. R. T. Seeley, Integro-differential operators on vector bundles, Trans. Amer. Math. Soc. 117 (1965), $167-204$.

Received December 19, 1976. Both authors supported in part by NSF Grant MPS 73-08593.

University of CAlifornia, Berkeley

Berkeley, CA 94720 




\section{Pacific Journal of Mathematics \\ Vol. 70, No. $1 \quad$ September, 1977}

William H. Barker, Noether's theorem for plane domains with hyperelliptic

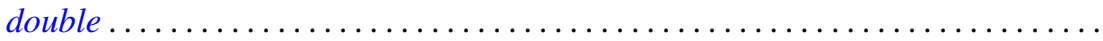

Michael James Beeson, Non-continuous dependence of surfaces of least area on the boundary curve ...................................... 11

Horst Behncke, Functions acting in weighted Orlicz algebras . . . . . . . . . . . . 19

Howard Edwin Bell, A commutativity study for periodic rings . . . . . . . . . . . 29

Peter Botta and Stephen J. Pierce, The preservers of any orthogonal group ....... 37

Douglas S. Bridges, The constructive Radon-Nikodým theorem ............. 51

James Dennis Brom, The theory of almost periodic functions in constructive

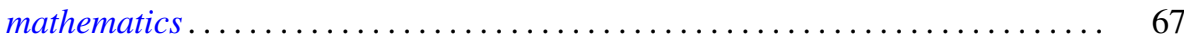

N. Burgoyne and C. Williamson, Semi-simple classes in Chevalley type groups ....

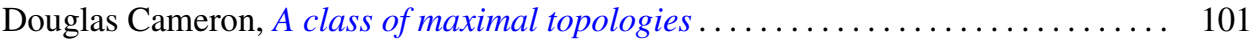

L. Carlitz, Enumeration of doubly up-down permutations . . . . . . . . . . . . . . 105

Paul Robert Chernoff, The quantum n-body problem and a theorem of

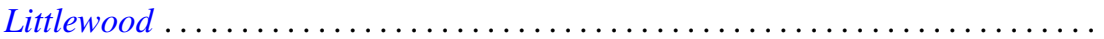

Jo-Ann Deborah Cohen, Locally bounded topologies on $F(X) \ldots \ldots \ldots \ldots \ldots \ldots$

Heinz Otto Cordes and Robert Colman McOwen, Remarks on singular elliptic

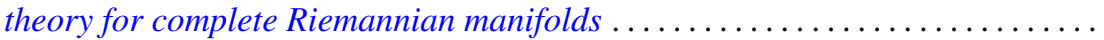

Micheal Neal Dyer, Correction to: "Rational homology and Whitehead

products"

Robert Fernholz, Factorization of Radonifying transformations

Lawrence Arthur Fialkow, A note on quasisimilarity. II ...... . .

Harvey Charles Greenwald, Lipschitz spaces of distributions on the surface of unit

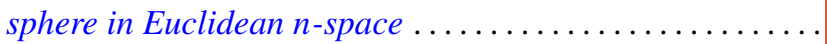

Albrecht Irle, On the measurability of conditional expectations

Tom (Roy Thomas Jr.) Jacob, Matrix transformations involving simple sequence

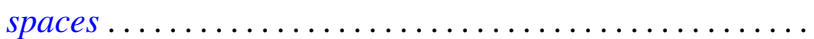

A. Katsaras, Continuous linear maps positive on increasing continuous

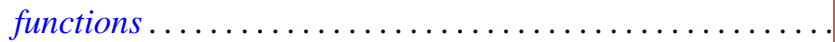

Kenneth Kunen and Judith Roitman, Attaining the spread at cardinals of cofinality

Lawrence Louis Larmore and Robert David Rigdon, Enumerating normal bundles

of immersions and embeddings of projective spaces ...... . .

Ch. G. Philos and V. A. Staïkos, Asymptotic properties of nonoscillatory solutions of differential equations with deviating argument .

Peter Michael Rosenthal and Ahmed Ramzy Sourour, On operator algebras containing cyclic Boolean algebras...

Polychronis Strantzalos, Strikt fast gleichgradig-stetige und eigentliche

Aktionen ...

Glenn Francis Webb, Exponential representation of solutions to an abstract

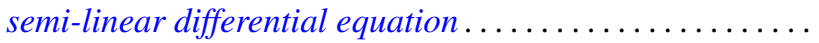

